\title{
Estimation of the Lower Bound Reliability of Latent Dimensions of Morphological Variables of Handball Players
}

\author{
Dragan Popovic, Evagelia Boli, Veroljub Stankovic, Milos Popovic, Jasna Popovic, \\ Vladimir Savic \\ Faculty of Sport and Physical Education, University of Pristina Temporarily Based in Leposavic, Leposavic, Serbia \\ Email: bogdragan.popovic@gmail.com
}

Received 5 January 2015; accepted 21 January 2016; published 27 January 2016

Copyright (C) 2015 by authors and OALib.

This work is licensed under the Creative Commons Attribution International License (CC BY). http://creativecommons.org/licenses/by/4.0/

(c) (i) Open Access

\begin{abstract}
Morphological characteristics describe a person on the basis of morphological (anthropometric) traits that are determined by anthropometric measurement techniques. The morphological structure of the entire psychosomatic status of a person means a system of basic anthropometric latent dimensions obtained from a series of manifest variables. In modern anthropometry, using factor analysis for data processing, four main factors or dimensionalities of the human body have been identified: longitudinal dimensionality, transversal dimensionality, circular dimensionality, and body mass and subcutaneous adipose tissue. As there are high correlations between these dimensions, constitutional types have been defined, making it possible to classify the human population by a particular morphological pattern.
\end{abstract}

\section{Keywords}

Morphological, Dimensions, Anthropometry, Structure, Psychosomatic Status

Subject Areas: Sports Science

\section{Introduction}

The concept of morphological characteristics implies the system of the structure of morphological dimensions bounded by a limited number of the manifest, directly measurable anthropometric measures.

In the course of physical growth and development, each body part follows a different curve, reaching its maximum at a different point of time. For this reason, the morphological structure of the body, which is based on mutual interactions between all anthropometric measures at different stages of development, can be different, i.e. individual morphological characteristics can at different points of time participate with different coefficients

How to cite this paper: Popovic, D., Boli, E., Stankovic, V., Popovic, M., Popovic, J. and Savic, V. (2016) Estimation of the Lower Bound Reliability of Latent Dimensions of Morphological Variables of Handball Players. Open Access Library Journal, 3: e1986. http://dx.doi.org/10.4236/oalib.1101986 
of participation in a particular morphological structure of the body.

However, the development of individual morphological characteristics is also largely governed by the structure of endogenous and exogenous factors which determine different physiological ages for different subjects in the same development period. In some morphological characteristics, especially those mostly affected by exogenous factors, variations within the population of the same chronological age can be very high.

On the basis of many to date studies carried out by applying the factorial approach and procedures [1] [2], it can be argued with certainty that a morphological space is essentially four-dimensional, which means that we have a model of the structure of morphological characteristics that consists of the four factors as follows:

L-longitudinal dimensionality of the skeleton, responsible for the bone growth in length;

T-transversal dimensionality of the skeleton, responsible for the bone growth in width;

V-body mass and volume, responsible for the total body mass and volume;

M-subcutaneous adipose tissue, responsible for the total amount of the body fat.

As the longitudinal dimensionality of the skeleton correlates most with the transversal dimensionality of the skeleton, and body mass and volume correlate with the subcutaneous adipose tissue, with respect to the gender and age, these factors are sometimes connected to form two factors: the dimensionality of the skeleton (longitudinal and transversal) and body volume (body mass and volume and subcutaneous adipose tissue).

Interaction factorial procedures were also used to isolate a general factor of growth (E) responsible for the total growth of all morphological characteristics.

A great number of studies in kinesiological anthropology deal with genetics and somatotypes. Namely, in the literature, the terms such as constitutiology (constitution, constitutional type, or body type or build) and somatotypology (somatotype) are often used in parallel.

Historically viewed, constitutiology is the oldest term used in anthropology to determine bodily individuality of man. Today, this term has a broader sense and denotes the wholeness of morphological and functional characteristics (inherited and acquired) that determines the features of the body's reactivity (intensity of reaction) and dynamics of ontogenesis. Also, in the literature, the concepts of general and individual (chromosomal, physical, biochemical, physiological, neurodynamic) constitution have been developed. Studying the body at different levels (micromorphological, macromorphological, biochemical, etc.), it can be observed that individual bodily constitutions have a common core (common line) connecting them in the form of genetic program which is realized during ontogenesis and under particular environmental conditions.

Somatotypology deals with classifying humans into constitutional types and such attempts date back virtually to the times of Hippocrates (2500 years ago) and his hypotheses about the existence of four structural elements of body type. Even though the systems for identifying the somatotype are numerous (their number equals the number of researchers dealing with this issue), body proportions, the amount of adipose tissue, the development level of the muscular system and skeleton are commonly taken for the criterion and classifications. In this regard, it should be highlighted that there is still no single realistic criterion, or model which can be used to select relevant morphological variables so as to reliably indicate, through an optimal condensation, that some morphological type exists.

On the grounds of different typologies, in recent studies the concept accepted by a large number of authors [3] starts from the hypothesis that each subject takes a single, relatively stable position on each of several multivariate continuous taxonomic variables. In order to test this hypothesis, it was needed to exclude the classical procedures of "cluster" analysis and apply the procedures based on the factorial or taxonomic model, the procedures belonging to the family of TAXOBOL algorithms, and thereby to achieve in an adequate way the typological goals of the study.

Taxonomic (T) approach and procedures. Despite the scarce number of studies which are still applied [3], [4] and regardless of the trends in constitutiology, the general morphological theoretical model is underpinned by the following taxons:

$\boldsymbol{\delta}$-skeletomorphy, responsible for skeleton longitudinality and partially bone width;

$\pi$-pycnomorphy, responsible for the prevalence of adipose tissue;

$\alpha$-athletomorphy, responsible for the size and amount of muscle mass and skeleton dimensionality;

$\varepsilon$-endomesomorphy, responsible for the prevalence of muscle and adipose tissue.

Although all the mentioned taxons are individually identified, they manifest themselves in an integrated manner and are, more or less, related to other characteristics of the anthropological status. 


\section{Methods}

\subsection{Sample of Subjects}

Selection of the sample of subjects was conditioned by the organizational and financial capabilities necessary for the implementation of the research process. A sufficient number of qualified and trained measurers as well as instrumentation and standardized conditions to realize the planned research were provided. The measurement was conducted on a sample representative of the whole Republic of Serbia. The sample was selected from the following clubs: RK Zeleznicar, RK Radnicki, RK Napredak, OK Mladi Radnik, OK Partizan, OK Ribnica, KK Konstantin, KK Radnicki, KK Zdravlje, FK Radnicki, and FK Napredak.

Besides the stated, the subjects were required to fulfill the following additional conditions:

- the subjects were male;

- the age of the subjects was defined based on chronological age, so that the research covered subjects from 18 to 22 plus - minus half a year;

- the subjects were registered players of the federal level competitions (the highest two levels of competition);

- the subjects regularly attended training as determined on the basis of records kept by coaches.

On the basis of the selected statistical and mathematical models and programs, objectives and hypotheses, the sample was decided to include 200 subjects.

In defining the population from which the sample of subjects was drawn, except the above, no other restrictions or stratification variables were applied.

\subsection{Sample of Variables}

For the evaluation of morphological characteristics of subjects, the researchers applied 20 anthropometric variables selected according to the International Biological Program (IBP) to cover the four-dimensional space defined as a longitudinal skeleton dimensionality, transversal skeleton dimensionality, volume and body mass and subcutaneous adipose tissue.

1) longitudinal skeleton dimensionality: body height (BOHEI), foot length (FOLEN), arm length (ARMLEN), leg length (LEGLEN), arm span (ARMSP);

2) transversal skeleton dimensionality: biacromial span (BIACSP), bicristal span (BICRSP), wrist diameter (WRID), elbow diameter (ELBD), knee diameter (KNED);

3) circular dimensionality and body weight: body weight (BODYW), chest circumference (CHESTC), upper arm circumference (UPARMC), forearm (FOARMC), upper leg circumference (UPLEGC);

4) subcutaneous adipose tissue: upper arm skin fold (UPARMSF), back skin fold (BACKSF), abdomen skin fold (ABDSF), armpit skin fold (ARMPSF), lower leg skin fold (LOLEGSF).

\subsection{Data Processing Methods}

The value of any research does not only depend on the sample of subjects and the sample of variables, that is, the basic information, but also on the procedures applied to transform and condense the information. Some scientific problems can be solved with the help of a larger number of different and sometimes equally valuable methods. However, different conclusions can be drawn from identical basic data and from the results obtained with different methods. That is why the problem of the choice of methods for data processing is rather complex.

To arrive at satisfactory scientific solutions, the study employed, first of all, correct, adequate, objective and comparable procedures appropriate for the character of the stated problem, enabling extraction and transformation of the appropriate dimensions as well as establishing regularities within the research area.

Taking the above into account, the researchers selected those procedures for the study that were considered to correspond to the nature of the problem and not to impose heavy restrictions on the basic information.

In the past years, a large number of researchers have misused their position and published an increasing number of quasi-scientific papers based primarily on mathematical artifacts. In addition, they use the existing statistical products, but they have never had any basic understanding of the logic of most multivariate models. Therefore, special attention of this paper is directed to statistical data processing and selection of those algorithms and programs that really have practical value.

Except for Mulaik's [5] famous textbook on factor analysis, which contains some information about estimation of reliability of principal components, and the work of Kaiser [6], who derived a method of Alpha factor analysis based on maximizing reliability of latent dimensions, it seems that producers of various methods of 
component and factor analyses as well as authors of books on this class of methods for latent structure analysis did not care about how much they could trust the real existence of the latent dimensions obtained with those methods. This fact also refers to the latent dimensions obtained by orthoblique transformation of principal components, a method that has become a standard procedure for analyzing latent structures among all those who have not acquired information about factor analysis as they used their fingers when reading seriously written texts in this area, or those who analyze their data using some of the commercially written statistical software packages, such as, but not exclusively, SPSS, CSS, Statistica, BMDP, and Statgraphics, not to mention other products whose popularity is much lower, but not always because they are significantly weaker than those abused today by some scientists and special sort of human beings called the "data processor" strain.

Indeed, one paper that proposes a competitive application of semi-orthogonal transformation of principal components in exploratory and confirmatory analyses of latent structures suggests application of the procedure for estimation of reliability of latent dimensions founded on Cronbach's strategy for generalizability evaluation. However, that procedure is as justifiable as the assumptions from which the Cronbach $\alpha$ coefficient has been derived, and even today it is called after him for unknown reasons, despite the fact that a totally identical measure, long before him, and virtually with the same assumptions, was proposed by [7], and in somewhat more simplified form described by [8], as well as by some other psychometricians who worked in the nascent stage of development of the measurement theory and in the age not caught in the PC revolution.

Therefore, the aim of this paper is to propose a measure for a lower bound to reliability of the latent dimensions obtained by the semi-orthogonal transformations of principal components.

All the data collected in this study were processed at the Multidisciplinary Research Center, through the system of data processing software programs DRSOFT developed by [2] [9] [10].

\section{Semi-Orthogonal Transformation of Principal Components}

Let $\mathbf{Z}$ be a standardized data matrix obtained by the description of some set $E$ of $n$ entities on some set $V$ of $\mathrm{m}$ quantitative, normally or at least elliptically distributed variables. Let $\boldsymbol{R}$ be an intercorrelation matrix of those variables. Assume that $\boldsymbol{R}$ is for certain a regular matrix and that the hypothesis that variables from $V$ have a spherical distribution can safely be rejected, and therefore, they are eigenvalues of the correlation matrix in population $P$ from which the sample $E$ has been extracted.

Let $\boldsymbol{U}^{2}=\left(\operatorname{diag} \boldsymbol{R}^{-1}\right)^{-1}$ be Guttman's [7] estimate of the unique variances of the variables from $\mathrm{V}$, and let $\lambda_{p}, p=$ $1, \cdots, m$ be eigenvalues of matrix $\boldsymbol{R}$. Let $c=\operatorname{trag}\left(I-U^{2}\right)$. Define scalar $k$ such that $\Sigma_{p}^{k} \lambda_{p}>c, \Sigma_{p}^{k-1} \lambda_{p}<c$.

Now, $k$ is the number of principal components of matrix $\boldsymbol{Z}$ determined according to Momirovic's PB criterion. Let $\Lambda=\left(\lambda_{p}\right) ; p=1, \cdots, k$ be a diagonal matrix of the first $k$ eigenvalues of matrix $\boldsymbol{R}$ and let $\boldsymbol{X}=\left(x_{p}\right), p=1, \cdots, k$ be a matrix of their associated eigenvectors scaled such that $\boldsymbol{X}^{t} \boldsymbol{X}=\boldsymbol{I}$. Let $\boldsymbol{T}$ be some orthonormal matrix such that it optimizes the function $\boldsymbol{X} \boldsymbol{T}=\boldsymbol{Q}=\left(\boldsymbol{q}_{p}\right) ; p(\boldsymbol{Q})=$ extremum, $\boldsymbol{T}^{t} \boldsymbol{T}=\boldsymbol{I}$, where $p(\boldsymbol{Q})$ is some parsimonious function, for instance, an ordinary Varimax function $\Sigma_{j}^{m} \Sigma_{p}^{k} q_{j p}^{4}-\Sigma_{p}^{k}\left(\Sigma_{j}^{m} q_{j p}^{2}\right)^{2}=$ maximum, where coefficients $q_{j p}$ are elements of matrix $\boldsymbol{Q}$ [6]. Now, the transformation of principal components, defined by vectors in the matrix $\boldsymbol{K}=$ $\boldsymbol{Z} \boldsymbol{X}$, into semi-orthogonal latent dimensions determined by type II of the orthoblique procedure [11], is defined by the operation $\boldsymbol{L}=\boldsymbol{K T}=\mathbf{Z X T}$. The covariance matrix of those dimensions is $\boldsymbol{C}=\boldsymbol{L}^{\prime} \mathbf{L} \boldsymbol{n}^{-1}=\mathbf{Q}^{\prime} \boldsymbol{R} \boldsymbol{Q} \boldsymbol{T}^{\boldsymbol{t}} \boldsymbol{\Lambda} \boldsymbol{T}$. Denote the matrix of their covariances by $\boldsymbol{S}^{2}=\left\{\left(s_{p}^{2}\right)\right\}=\operatorname{diag} \boldsymbol{C}$. If the latent dimensions are standardized by the operation $\boldsymbol{D}=\boldsymbol{L} \boldsymbol{S}^{-1}$, their intercorrelations will be in the matrix $\boldsymbol{M}=\boldsymbol{D}^{t} \boldsymbol{D} n^{-1}=\boldsymbol{S}^{-1} \boldsymbol{T}^{t} \boldsymbol{\Lambda} \boldsymbol{T}^{-1}$; note that neither $\boldsymbol{C}$, and therefore nor $\boldsymbol{M}$, can be the diagonal matrices, so thus obtained latent dimensions are not orthogonal in the space of $\mathrm{E}$ entity. The matrix of correlations between the variables from $\mathrm{V}$ and latent variables, commonly referred to as a factor structure matrix, will be $\boldsymbol{F}=Z^{t} \boldsymbol{D} n^{-1}=\boldsymbol{R} \boldsymbol{X} \boldsymbol{T} \boldsymbol{S}^{-1}=\boldsymbol{X} \boldsymbol{\Lambda} \boldsymbol{T} \boldsymbol{S}^{-1}$; and since the elements of matrix $\boldsymbol{F}$ are orthogonal projections of the vectors from $\boldsymbol{Z}$ onto the vectors from $\boldsymbol{D}$, the coordinates of those vectors in the space spanned by the vectors from $\boldsymbol{D}$ are elements of the matrix $\boldsymbol{A}=\boldsymbol{F} \boldsymbol{M}^{-1}=\boldsymbol{X} \boldsymbol{T S}$. However, as $\boldsymbol{A}^{t} \boldsymbol{A}=\boldsymbol{S}^{2}$, the latent dimensions obtained through this procedure are orthogonal in the space spanned by the vectors of variables from $Z$; quadratic norms of the vectors of these dimensions in the space of the variables equal the variances of these dimensions.

\section{Estimation of the Lower Bound of Reliability of Latent Dimensions}

For simplicity and clear algebraic and geometric meaning as well as latent dimensions and identification struc- 
tures associated with these dimensions, reliability of the latent dimensions obtained by orthoblique transformation of principal components can be determined clearly and unequivocally.

Let $\boldsymbol{G}=\left(g_{i j}\right) ; i=1, \cdots, n ; j=1, \cdots, m$ be some tolerably unknown matrix of errors of the estimate in describing the set $\mathrm{E}$ on the set $\mathrm{V}$. Then the matrix of true results of entities from $\mathrm{E}$ on the variables from $\mathrm{V}$ will be $\boldsymbol{Y}=\mathbf{Z}-$ $\boldsymbol{G}$. If we assume, in accordance with the classical measurement theory, that matrix $\boldsymbol{G}$ is such that $\boldsymbol{Y}^{t} \boldsymbol{G}=\mathbf{0}$ and $\boldsymbol{G}^{t} \boldsymbol{G} n^{-1}=\boldsymbol{E}^{2}=\left(e_{j j}^{2}\right)$, where $\boldsymbol{E}^{2}$ is a diagonal matrix, the covariance matrix of real results will be $\boldsymbol{H}=\mathbf{Y}^{t} \mathbf{Y} n^{-1}=\boldsymbol{R}$ $-\boldsymbol{E}^{2}$ if $\boldsymbol{R}=\mathbf{Z}^{t} \mathbf{Z n}^{-1}$, the intercorrelation matrix of variables from $\mathrm{V}$ is defined on the set $\mathrm{E}$.

Assume that reliability coefficients of variables from $\mathrm{V}$ are known; let $\boldsymbol{P}$ be a diagonal matrix whose elements $\rho_{j}$ are those reliability coefficients. Then the variances of errors of the estimate for standardized results on the variables from $\mathrm{V}$ will be exactly the elements of the matrix $\boldsymbol{E}^{2}=\boldsymbol{I}-\boldsymbol{P}$. Now, the true values of the latent dimensions will be the elements of the matrix $\boldsymbol{\Gamma}=(\boldsymbol{Z}-\boldsymbol{G}) \boldsymbol{Q}$ along with the covariance matrix $\boldsymbol{\Omega}=\Gamma^{t} \Gamma n^{-1}=\boldsymbol{Q}^{t} \mathbf{H} \boldsymbol{Q}=$ $\boldsymbol{Q}^{t} \boldsymbol{R} \boldsymbol{Q}-\boldsymbol{Q}^{t} \boldsymbol{E}^{2} \boldsymbol{Q}=\left(\omega_{p q}\right)$. Accordingly, the true variances of the latent dimensions will be the diagonal elements of the matrix $\Omega$; denote those elements by $\omega_{p}^{2}$. Based on the formal definition of reliability coefficients of a variable, $\rho=\delta_{t}^{2} / \delta^{2}$ where $\delta_{t}^{2}$ is the true variance of some variable and $\delta^{2}$ is the total variance of that variable, that is, the variance which includes the error variance, reliability coefficients of latent dimensions will be $\gamma_{p}$ $\gamma_{p}=\boldsymbol{w}_{p}^{2} / \mathbf{s}_{p}^{2}=1-\left(\boldsymbol{q}_{p}^{t} \boldsymbol{E}^{2} \boldsymbol{q}_{p}\right)\left(\boldsymbol{q}_{p}^{t} \boldsymbol{R} \boldsymbol{q}\right)^{-1} \quad p=1, \cdots, k$ if reliability coefficients of the variables from which those dimensions are derived are known.

Proposition 1. Coefficients $\gamma_{p}$ vary in the range of (0.1) and can assume the value of 1 if and only if $\boldsymbol{P}=\boldsymbol{I}$, so if all variables have been estimated without errors, and the value is 0 if and only if both $\boldsymbol{P}=\mathbf{0}$ and $\boldsymbol{R}=\boldsymbol{I}$, i.e. if the total variance of all the variables consists only of the variance of errors of the estimate, the variables from $\mathrm{V}$ have a spherical normal distribution.

Proof: If the total variance of each variable from some set of variables consists only of a variance of errors of the estimate, then necessarily that $\boldsymbol{E}^{2}=\boldsymbol{I}$ and $\boldsymbol{R}=\boldsymbol{I}$, and therefore, all coefficients $\gamma_{p}$ equal zero. The first part of the Proposition is obvious from the definition of coefficients $\gamma_{p}$; this means that reliability of each latent dimension, regardless of the manner of determining that latent dimension, equals1 if the variables, from which that dimension has been derived, are estimated without errors.

However, the matrix of reliability coefficients $\boldsymbol{P}=\left(\rho_{j}\right)$ is often unknown, therefore the variance matrix of errors of the estimate $\boldsymbol{E}^{2}$ is unknown. But if variables from $\mathrm{V}$ are selected so as to represent some universe of variables $U$ with the same field of meaning, the upper bound for the variances of errors of the estimate is defined by the elements of matrix $\boldsymbol{U}^{2}$ [7], hence by the unique variances of those variables. Accordingly, in that case, the lower bound of reliability of latent dimensions can be estimated by the coefficients

$\boldsymbol{\beta}_{p}=1-\left(\boldsymbol{q}_{p}^{t} \boldsymbol{U}^{2} \boldsymbol{q}_{p}\right)\left(\boldsymbol{q}_{p}^{t} \boldsymbol{R} \boldsymbol{q}_{p}\right)^{-1} \quad p=1, \cdots, k$ which have been derived through the procedure identical to that used to derive coefficients $\gamma_{p}$ with the definition $\boldsymbol{E}^{2}=\boldsymbol{U}^{2}$, that is, in the same way as Guttman derived his $\lambda_{6}$ estimate.

\section{Structure of Morphological Dimensions of Handball Players}

Morphological characteristics of the anthropological status of a person imply a certain system of basic anthropometric latent dimensions. Today it is impossible to imagine any serious planning of a motor activity without knowing the morphological structure of its influence on this activity as well as the influence of the activity on the development of morphological characteristics.

Morphological characteristics and somatotypic features have long drawn the attention of many researchers from the need to determine principles of development in general and especially development of an athlete's organism, contribution of these characteristics in the realization of certain motor skills and habits.

Measures of longitudinal dimensionality take a special place among morphological characteristics, and the body height and large dimensionality of the ends of the limbs are significant and particularly important; the span of the open hand from the tip of the thumb to the tip of the little finger should specially be emphasized. The size of this span conditions possibility of holding a ball with an overhand grip, and only an overhand grip makes it possible to master almost all and especially the most important elements of the game techniques in attack.

Furthermore, handball is a game that besides the physical characteristics of the player, requires a certain degree of intelligence and imagination as well as the ability to find the best solutions making quick decisions in uncertain situations.

The starting matrix to identify the structure in component analysis is a complete matrix of intercorrelations. 
From thus obtained intercorrelation matrix, using principal component analysis, $75.93 \%$ of variability of the applied system of variables was explained. By using Momirovic's $\beta_{6}$ criterion, four principle components whose characteristic roots met the given condition were obtained (Table 1).

The first principal component with the characteristic root of 9.03 and variance of $48.99 \%$ was explained by all the variables of longitudinal, transversal and circular dimensionalities, as well as two variables for estimating adiposity of the back and armpits of handball players (BACKSF and ARMPSF). Based on the high correlations which the mentioned variables have with the first principal component, it can be assumed with certainty that it acts as a general factor of growth and development of young handball players.

The second principal component explains $14.12 \%$ of the common variance. It represents a dual factor of measurements for estimation of subcutaneous adipose tissue of the upper arm (UPARMSF) and subcutaneous adipose tissue of the lower leg (LOLEGSF), on the basis of which it can be concluded that this ballast tissue represents a significant but not dominant characteristic of the selected young handball players.

Table 1. Matrix of principal components of anthropometric variables of handball players.

\begin{tabular}{|c|c|c|c|c|c|}
\hline & FAC1 & FAC2 & FAC3 & FAC4 & $h^{2}$ \\
\hline BOHEI-body height & 0.86 & -0.36 & 0.24 & 0.00 & 0.99 \\
\hline FOLEN-foot length & 0.83 & 0.29 & 0.15 & 0.01 & 0.79 \\
\hline ARMLEN-arm length & 0.75 & 0.39 & 0.26 & 0.03 & 0.79 \\
\hline LEGLEN-leg lenth & 0.79 & 0.39 & 0.33 & 0.01 & 0.88 \\
\hline ARMSP-arm span & 0.89 & 0.38 & 0.17 & 0.00 & 0.93 \\
\hline BIACSP-biacromial span & 0.83 & 0.08 & 0.08 & 0.11 & 0.69 \\
\hline BICRSP-bicristal span & 0.82 & 0.08 & 0.05 & 0.08 & 0.68 \\
\hline WRID-wrist diameter & 0.76 & 0.33 & 0.19 & 0.10 & 0.73 \\
\hline ELBD-elbow diameter & 0.66 & 0.30 & 0.19 & 0.30 & 0.68 \\
\hline KNED-knee diameter & 0.86 & 0.15 & 0.21 & 0.07 & 0.98 \\
\hline BODYW-body weight & 0.18 & 0.01 & 0.51 & 0.49 & 0.55 \\
\hline CHESTC-chest circumference & 0.87 & 0.12 & 0.30 & 0.04 & 0.87 \\
\hline UPARMC-upper arm circumference & 0.86 & 0.24 & 0.28 & 0.02 & 0.88 \\
\hline FOARMC-forearm & 0.87 & 0.11 & 0.35 & 0.00 & 0.89 \\
\hline UPLEGC-upper leg circumference & 0.56 & 0.35 & 0.00 & 0.11 & 0.47 \\
\hline UPARMSF-upper arm skin fold & 0.57 & 0.63 & 0.17 & 0.16 & 0.77 \\
\hline BACKSF-back skin fold & 0.68 & 0.59 & 0.00 & 0.03 & 0.83 \\
\hline ABDSF-abdomen skin fold & -0.01 & 0.02 & 0.21 & 0.76 & 0.64 \\
\hline ARMPSF-armpit skin fold & 0.50 & 0.47 & 0.46 & 0.19 & 0.65 \\
\hline LOLEGSF-lower leg skin fold & 0.50 & 0.63 & 0.34 & 0.12 & 0.79 \\
\hline Charact. roots & 9.03 & 4.11 & 2.44 & 1.14 & \\
\hline$\%$ Variance & 48.99 & 14.12 & 0.71 & 0.11 & \\
\hline Cumulat. \% & 48.99 & 62.11 & 9.82 & 75.93 & \\
\hline
\end{tabular}


The third and fourth principal components also represent single factors. The third principle component is defined by the body mass (BODYW) and the fourth—by the abdomen skin fold (ABDSF).

The communality value for all the variables is satisfactory.

To obtain a parsimonious structure in order to make such a clear structure even more simplified, the obtained initial coordinate system is transformed into a slanting oblimin position, after which the same number of factors is retained. Because the method used for the transformation provides a total of three matrices: a matrix of parallel projections of the variables onto the factors (Table 2), a matrix of orthogonal projections of the variables onto the factors (Table 3 ) and a matrix of intercorrelations of the obtained factors, all the three matrices are interpreted simultaneously.

The first oblimin factor has the largest projections with the variables of longitudinal and transversal dimensionalities. It can, without any doubt, be interpreted as a general factor of the skeleton growth of the selected handball players.

The second oblimin factor is defined by the variables for the estimation of adipose tissue of the selected handball players. The highest saturation of this factor is provided by the variables of the lower leg skin fold

Table 2. Pattern matrix of anthropometric variables of handball players.

\begin{tabular}{|c|c|c|c|c|}
\hline & \multicolumn{4}{|c|}{ Components } \\
\hline & OBL1 & OBL2 & OBL3 & OBL4 \\
\hline BOHEI-body height & 0.98 & 0.02 & 0.07 & 0.07 \\
\hline FOLEN-foot length & 0.86 & 0.04 & 0.00 & 0.01 \\
\hline ARMLEN-arm length & 0.94 & 0.08 & 0.10 & 0.09 \\
\hline LEGLEN-leg length & 0.99 & 0.02 & 0.17 & 0.10 \\
\hline ARMSP-arm span & 0.97 & 0.01 & 0.01 & 0.01 \\
\hline BIACSP-biacromial span & 0.58 & 0.11 & 0.29 & 0.00 \\
\hline BICRSP-bicristal span & 0.69 & 0.23 & 0.14 & 0.03 \\
\hline WRID-wrist diameter & 0.69 & 0.16 & 0.24 & 0.29 \\
\hline ELBD-elbow diameter & 0.60 & 0.06 & 0.12 & 0.49 \\
\hline KNED-knee diameter & 0.45 & 0.38 & 0.34 & 0.27 \\
\hline BODYW-body weight & 0.19 & 0.09 & 0.76 & 0.12 \\
\hline CHESTC-chest circumference & 0.34 & 0.35 & 0.48 & 0.17 \\
\hline UPARMC-upper arm circumference & 0.24 & 0.46 & 0.47 & 0.18 \\
\hline FOARMC-forearm & 0.34 & 0.32 & 0.50 & 0.20 \\
\hline UPLEGC-upper leg circumference & 0.09 & 0.56 & 0.20 & 0.05 \\
\hline UPARMSF-upper arm skin fold & 0.07 & 0.89 & 0.07 & 0.12 \\
\hline BACKSF-back skin fold & 0.04 & 0.86 & 0.16 & 0.08 \\
\hline ABDSF-abdomen skin fold & 0.10 & 0.09 & 0.19 & 0.74 \\
\hline ARMPSF-armpit skin fold & 0.10 & 0.78 & 0.08 & 0.28 \\
\hline LOLEGSF-lower leg skin fold & 0.08 & 0.99 & 0.21 & 0.00 \\
\hline
\end{tabular}


Table 3. Structure matrix of anthropometric variables of handball players.

\begin{tabular}{|c|c|c|c|c|}
\hline & OBL1 & OBL2 & OBL3 & OBL4 \\
\hline BOHEI-body height & 0.98 & 0.38 & 0.25 & 0.12 \\
\hline FOLEN-foot length & 0.87 & 0.47 & 0.31 & 0.14 \\
\hline ARMLEN-arm length & 0.87 & 0.35 & 0.19 & 0.06 \\
\hline LEGLEN-leg length & 0.90 & 0.35 & 0.15 & 0.05 \\
\hline ARMSP-arm span & 0.96 & 0.38 & 0.32 & 0.15 \\
\hline BIACSP-biacromial span & 0.72 & 0.42 & 0.59 & 0.15 \\
\hline BICRSP-bicristal span & 0.77 & 0.52 & 0.42 & 0.11 \\
\hline WRID-wrist diameter & 0.77 & 0.26 & 0.48 & 0.37 \\
\hline ELBD-elbow diameter & 0.68 & 0.22 & 0.35 & 0.51 \\
\hline KNED-knee diameter & 0.66 & 0.65 & 0.58 & 0.38 \\
\hline BODYW-body weight & 0.02 & 0.01 & 0.68 & 0.16 \\
\hline CHESTC-chest circumference & 0.61 & 0.63 & 0.70 & 0.32 \\
\hline UPARMC-upper arm circumference & 0.60 & 0.68 & 0.71 & 0.36 \\
\hline FOARMC-forearm & 0.68 & 0.61 & 0.72 & 0.36 \\
\hline UPLEGC-upper leg circumference & 0.38 & 0.64 & 0.36 & 0.05 \\
\hline UPARMSF-upper arm skin fold & 0.29 & 0.86 & 0.12 & 0.23 \\
\hline BACKSF-back skin fold & 0.38 & 0.88 & 0.36 & 0.18 \\
\hline ABDSF-abdomen skin fold & 0.02 & 0.03 & 0.15 & 0.76 \\
\hline ARMPSF-armpit skin fold & 0.33 & 0.74 & 0.11 & 0.19 \\
\hline LOLEGSF-lower leg skin fold & 0.26 & 0.85 & 0.09 & 0.07 \\
\hline
\end{tabular}

(LOLEGSF, the upper arm skin fold (UPERMSF), the back skin fold (BACKSF), the armpit skin fold (ARMPSF) as well as a variable for estimating the circular dimensionality of the skeleton-the upper leg circumference (UPLEGC). It is a factor that is not generated through a multi-year training process because the sample of subjects is represented by young handball players, but it is the consequence of endogenous influence. This factor can be defined as a factor of subcutaneous adipose tissue or endomorph.

The third oblimin factor has a simple structure and can be interpreted in a similar way as in many previous studies on this and similar samples of athletes [12]. It is a factor of the volume and mass of the body, that is, the variables which play an important role in the percentage share in the total body mass.

The fourth oblimin factor is a single factor of the abdomen skin fold and here it probably refers to a product of hyper factorization.

The first three factors have statistically significant correlations between the isolated factors, which is logical because the selected young handball players have slightly higher accumulation of subcutaneous adipose tissue and voluminosity that are correlated with the general growth factor.

Some morphological characteristics of handball players have a significant degree of correlation with success in handball, or in a handball game. In a series of studies, it was determined that tall handball players with longer arms and legs have a certain advantage over shorter handball players or handball players with shorter limbs [12]. 
Their movements in the game are more efficient. Handball players with a larger amount of adipose tissue are more superior in complex motor structures. The amount of influence of a specific morphological structure on success in a handball game should be found by determining the degree of correlation between the complete battery of anthropometric tests with success in the game. It can be expected that the length of the arms, legs, feet, biacromial span, weight, circumference of the chest, upper arm, forearm and upper leg, the width of the hand and foot, wrist diameter, as well as skin folds of the abdomen and back have more important correlations with success in the game than other measures. It necessarily follows from all these that a structure which corresponds to the structure of handball players has been obtained in the morphological space.

\section{Conclusions}

The research was conducted to determine the structure of morphological dimensions of athletes who are engaged in handball. In order to determine the structure of the treated morphological dimensions, 200 handball players were examined.

For estimation of morphological characteristics of the subjects, 20 anthropometric variables, selected according to the International Biological Program (IBP), were applied to cover the four-dimensional space defined as a longitudinal skeletal dimensionality, transversal skeletal dimensionality, volume and mass of the body, and subcutaneous adipose tissue.

All the data in this study were processed at the Multidisciplinary Research Center of the Faculty of Sport and Physical Education, University of Pristina through the system of data processing software programs DRSOFT developed by [2] [9] and [10].

The algorithm and program realized in this study are fully presented, and the results of this program are analyzed.

To determine the latent structure of handball players in a morphological space, a method of component factor analysis was applied.

By using Momirovic's $\beta_{6}$ criterion in a morphological space, four significant components whose characteristic roots met the given condition were obtained (Table 1).

The first principal component with the characteristic root of 9.03 and variance of $48.99 \%$ was explained by all the variables of longitudinal, transversal and circular dimensions, as well as two variables for estimating adiposity of the back and armpits of handball players (BACKSF and ARMPSF). Based on the high correlations which the mentioned variables have with the first principal component, it can be assumed with certainty that it acts as a general factor of growth and development of young handball players.

The second principal component explains a total of $14.12 \%$ of the common variance. It is a dual factor of measurement for evaluation of subcutaneous adipose tissue of the upper arm (UPARMSF) and subcutaneous adipose tissue of the lower leg (LOLEGSF), on the basis of which it can be concluded that this ballast tissue represents a significant but not dominant characteristic of the selected young handball players.

The third and fourth principal components also represent single factors. The third principal component is defined by the body mass (BODYW), and fourth—by the abdomen skin fold (ABDSF) (Table 4).

The communality value for all the variables is satisfactory.

Some morphological characteristics of handball players have a significant degree of correlation with success in handball, or in a handball game. In a series of studies, it was determined that tall handball players with longer arms and legs have a certain advantage over shorter handball players or handball players with shorter limbs [12].

Table 4. Intercorrelations of oblimin factors.

\begin{tabular}{|c|c|c|c|c|}
\hline & OOBL1 & OBL2 & OBL3 & OBL4 \\
\hline OBL1 & 10.00 & 0.41 & 0.34 & 0.18 \\
\hline OBL2 & 0.41 & 10.00 & 0.23 & 0.10 \\
\hline OBL3 & 0.34 & 0.23 & 10.00 & 0.09 \\
\hline OBL4 & 0.18 & 0.10 & 0.09 & 10.00 \\
\hline
\end{tabular}


Their movements in the game are more efficient. Handball players with a larger amount of adipose tissue are more superior in complex motor structures. The value of the influence of a specific morphological structure on the success in a handball game should be found by determining the degree of correlation between the complete battery of anthropometric tests and success in the game. It can be expected that the length of the arms, legs, feet, biacromial span, body weight, circumference of the chest, upper arm, forearm and upper leg, the width of the hand and foot, wrist diameter, and skin folds of the abdomen and back have more significant correlations with success in the game than other measures. It necessarily follows from all these that a structure which corresponds to the structure of handball players has been obtained in the morphological space.

\section{References}

[1] Momirovic, K. (1969) Factor Structure of Anthropometric Variables. Institute of Kinesiology, College of Physical Education, Zagreb.

[2] Popovic, D. (1993a) Programs and Subprograms for the Analysis of Quantitative Modifications. Faculty of Physical Education, Multidisciplinary Research Center, Pristina, Serbia.

[3] Popovic, D. and Boli, E. (2002) Relations between Suppleness, Morphological Characteristics and Motor Abilities of Boys. 7th Annual Congress of the European College of Sport Science, Cologne, 24-28 July 2002, 504.

[4] Hosek-Momirovic, A. (1981) Relationship between Morphological Taxons and Manifest and Latent Dimensions of Coordination. Kinesiology, 4, 5-204.

[5] Mulaik, S.A. (1972) The Foundations of Factor Analysis. McGraw-Hill, New York.

[6] Kaiser, H.F. (1958) The Varimax Criterion for Analytic Rotation in Factor Analysis. Psychometrika, 23, 187-200. http://dx.doi.org/10.1007/BF02289233

[7] Guttman, L. (1945) Basis for Test-Retest Reliability Analysis. Psychometrika, 10, 255-282. http://dx.doi.org/10.1007/BF02288892

[8] Momirovic, D., Wolf, B. and Popovic, D. (1999) The Introduction to the Theory of Measurement and Internal Metric Properties of Composite Measuring Instruments. Faculty of Physical Education, Multidisciplinary Research Center, Pristina, Serbia.

[9] Popovic, D. (1980) Research Methodology in Physical Education. University of Nis, Nis, Serbia.

[10] Momirovic, K. and Popovic, D. (2003) Construction and Application of Taxonomy Neural Networks. Faculty of Physical Education, Multidisciplinary Research Center, Leposavic, Serbia.

[11] Harris, C.W. and Kaiser, H.F. (1964) Oblique Factor Analytic Solutions by Orthogonal Transformations. Psychometrika, 29, 347-362. http://dx.doi.org/10.1007/BF02289601

[12] Popovic, D., Stanković, V. and Ilić, S. (1998) The Structure of Morphological Characteristics of Young Handball Players. 6th International Congress on Physical Education and Sport, Komotini, 148. 